\title{
Aortic valve: To buy or not to buy?
}

Jihoon Kim, MD, PhD, ${ }^{a}$ and Jae Won Lee, MD, $\mathrm{PhD}^{\mathrm{b}}$

\footnotetext{
From the ${ }^{\mathrm{a}}$ Department of Thoracic and Cardiovascular Surgery, Kangnam Sacred Heart Hospital, Hallym University Medical Center, Hallym University College of Medicine, Seoul, Republic of Korea; and ${ }^{\mathrm{b}}$ Department of Thoracic and Cardiovascular Surgery, Asan Medical Center, University of Ulsan College of Medicine, Seoul, Republic of Korea.

Disclosures: Authors have nothing to disclose with regard to commercial support.

Received for publication Feb 5, 2018; accepted for publication Feb 10, 2018; available ahead of print March 16, 2018.

Address for reprints: Jae Won Lee, MD, PhD, Department of Thoracic and Cardiovascular Surgery, Asan Medical Center, University of Ulsan College of Medicine, 88, Olympic-Ro 43-Gil, Songpa-Gu, Seoul 05505, Republic of Korea (E-mail: jwlee@amc.seoul.kr).

J Thorac Cardiovasc Surg 2018;155:2388-9 $0022-5223 / \$ 36.00$

Copyright (c) 2018 Published by Elsevier Inc. on behalf of The American Association for Thoracic Surgery https://doi.org/10.1016/j.jtcvs.2018.02.031
}

With understanding of the anatomy and pathophysiology, surgical aortic valve repair techniques have undergone improvement during the past several decades. ${ }^{1}$ The techniques, however, are mostly for aortic regurgitation, and thus, most patients with aortic stenosis are treated with surgical aortic valve replacement (SAVR) or transcatheter aortic valve replacement (TAVR). Severely calcified, thickened, and deformed leaflets are deemed basically nonrepairable, so surgeons proceed to remove them and reconstruct them.

In this context, $\mathrm{Al}$ Halees and colleagues ${ }^{2}$ reported their up-to 16-year follow-up data of aortic valve reconstruction with pericardium in 2005 . They used molds of varying sizes to produce neoleaflets with pericardium. ${ }^{3}$ Xenopericardium and glutaraldehyde-treated autologous pericardium are used for the reconstruction, and the authors concluded that aortic valve reconstruction is feasible, and both of the pericardia are useful as reconstruction material.

Ozaki and colleagues ${ }^{4}$ first published their initial experience on the aortic valve neo-cuspidization (AVNeo) with glutaraldehyde-treated autologous pericardium in 2011. They made neoleaflets with a large coaptation area after measuring respective intercommissural distances with their "self-developed aortic valve plasty system." The theoretical basis of AVNeo is to obtain large coaptation area by oversizing and thus alleviating stress on each cusp, which results in increased longevity of the leaflets.

In this issue of the Journal, Ozaki and colleagues ${ }^{5}$ reported the midterm result of the AVNeo in 850 patients, with the longest follow-up being 118 months. AVNeo procedures were performed on virtually all the diagnoses of the aortic valve diseases, and the clinical outcomes were acceptable: in-hospital mortality $(1.8 \%)$, actuarial freedom from death $(85.9 \%)$, cumulative incidences of reoperation $(4.2 \%)$, and recurrent moderate aortic regurgitation or greater $(7.3 \%)$. On echocardiography, peak pressure gradients were on average $19.5 \pm 10.3 \mathrm{~mm} \mathrm{Hg} 1$ week after surgery and $15.2 \pm 6.3 \mathrm{~mm} \mathrm{Hg}$ at 8 years. results. considered. warranted.

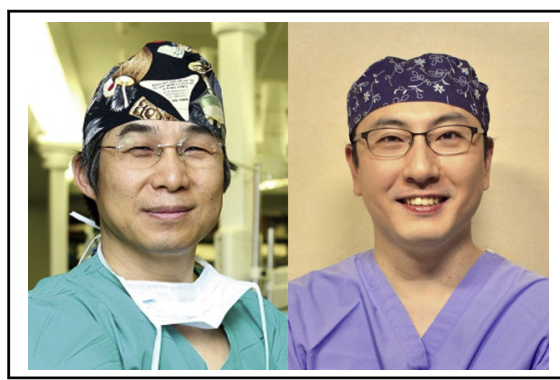

Jae Won Lee, MD, PhD, and Jihoon Kim, MD, PhD

\section{Central Message}

Reconstruction of the aortic valve leaflets requires compatible material with proper design. Both of the components have yet to be identified

See Article page 2379.

AVNeo is attractive because of its low cost, universal indications without any limit in annulus size (possible pediatric application) nor any need for anticoagulation, and its potentially excellent hemodynamics. In contrast, we hesitate to perform the AVNeo because of its long aortic crossclamp time (106.8 \pm 27.5 minutes), complex procedure, unidentified flow dynamics, and lack of any long-term

There are 2 components in reconstructing the valve leaflets; material and design. First, there are several materials that can be used as leaflets; autologous pericardium treated with glutaraldehyde solution with various concentrations and treatment time, xenopericardium (ie, bovine and equine), and synthetic patches. The best material is yet to be identified. Second, the design of the new aortic valve could be a replica of a healthy aortic valve or a modified shape that might yield a superior hemodynamic profile with durability. Furthermore, in this era of TAVR, the compatibility with TAVR should be

The authors should be commended for their efforts to develop a universal solution for aortic valvular diseases. However, numerous prosthetic tissue valves are in development with outstanding hemodynamics, prolonged longevity, and ease of implantation. AVNeo must prove its superiority over SAVR and TAVR to be accepted as the standard treatment modality; therefore, subsequent long-term follow-up studies including hemodynamics are 


\section{References}

1. Aicher D, Schäfers H-J. Aortic valve repair-current status, indications, and outcomes. Semin Thorac Cardiovasc Surg. 2012;24:195-201.

2. Al Halees Z, Al Shahid M, Al Sanei A, Sallehuddin A, Duran C. Up to 16 years follow-up of aortic valve reconstruction with pericardium: a stentless readily available cheap valve? Eur J Cardiothorac Surg. 2005;28:200-5; discussion 205.

3. Duran CM, Gometza B, Kumar N, Gallo R, Martin-Duran R. Aortic valve replacement with freehand autologous pericardium. J Thorac Cardiovasc Surg. 1995;110:511-6.

4. Ozaki S, Kawase I, Yamashita H, Uchida S, Nozawa Y, Matsuyama T, et al. Aortic valve reconstruction using self-developed aortic valve plasty system in aortic valve disease. Interact Cardiovasc Thorac Surg. 2011;12: 550-3.

5. Ozaki S, Kawase I, Yamashita H, Uchida S, Takatoh M, Kiyohara N Midterm outcomes after aortic valve neocuspidization with glutaraldehydetreated autologous pericardium. J Thorac Cardiovasc Surg. 2018;155: 2379-87. 